\title{
A Study on Glycosylated Haemoglobin Values at 6 - 12 Weeks of Pregnancy as an Early Predictor of Gestational Diabetes Mellitus
}

\author{
Staeny Rex ${ }^{1}$, Preet Agarwal'2, Sarmishta Murugesan ${ }^{3}$, Rajeshwari K.S. ${ }^{4}$ \\ ${ }^{1,2,34}$ Department of Obstetrics \& Gynaecology, Sri Ramachandra Institute of Higher \\ Education and Research, Porur, Chennai, Tamil Nadu, India.
}

\section{ABSTRACT}

\section{BACKGROUND}

Gestational diabetes mellitus (GDM) is a major health problem arising due to insulin resistance in pregnant women. It is associated with multiple maternal complications which may cause end organ failure, complicated labour and delivery and thereby increasing the maternal morbidity and mortality. The foetus is also at risk for problems beginning from in utero and extending into the neonatal period and adult life. We wanted to correlate first trimester HbA1c values with the subsequent development of gestational diabetes mellitus and identify if glycosylated haemoglobin can be used as an adjunct with other screening methods.

\section{METHODS}

It is a prospective cohort study. This study was conducted from August 2015 to August 2017. All women of gestational age 6 to 12 weeks who came to Sri Ramachandra University outpatient department were taken for this study. Informed consent was obtained and a detailed history taking was done as per proforma. Along with routine antenatal investigations, Glycosylated haemoglobin (HbA1c) was also done. Oral Glucose Tolerance Test was to be performed at $24-28$ weeks as per the World Health Organisation -with 75gm criteria and the results were tabulated and analysed to know the significance of Glycosylated haemoglobin (HbA1c) in the outcome of gestational diabetes mellitus. Further a fasting and postprandial blood sugar was done in the $3^{\text {rd }}$ trimester for all patients as a secondary screening tool for gestational diabetes mellitus. Secondary outcomes of obstetric and neonatal complications were also studied.

\section{RESULTS}

In the present study a total of 323 patients were screened for HbA1c during the first trimester and followed till delivery. Only $21.9 \%$ developed gestational diabetes mellitus. Elevated Glycosylated haemoglobin (HbA1c) value of 5.7 - $6.4 \%$ was seen in $5.9 \%$.

\section{CONCLUSIONS}

Most of the patients with elevated Glycosylated Haemoglobin in the first trimester had high chance of developing gestational diabetes mellitus. Glycosylated haemoglobin is indeed a simple way of screening, but its solitary use remains controversial and hence could possibly be tried as an adjunct with other screening methods.

\section{KEY WORDS}

Glycosylated Haemoglobin, Gestational Diabetes Mellitus, Glycaemic Control, Maternal and Foetal Morbidity and Mortality
Corresponding Author: Dr. Preet Agarwal, Sri Ramachandra Institute of Higher Education and Research, Porur, Chennai, Tamil Nadu, India. E-mail: sarmishta83@yahoo.co.in

DOI: $10.14260 /$ jemds/2021/508

How to Cite This Article:

Rex S, Agarwal P, Murugesan S, et al. A study on glycosylated haemoglobin values at 6 - 12 weeks of pregnancy as an early predictor of gestational diabetes mellitus. $J$ Evolution Med Dent Sci 2021;10(31): 2485-2489, DOI:

$10.14260 /$ jemds $/ 2021 / 508$

Submission 20-01-2021,

Peer Review 15-05-2021

Acceptance 21-05-2021,

Published 02-08-2021.

Copyright (C) 2021Staeny Rex et al. This is an open access article distributed under Creative Commons Attribution License [Attribution 4.0 International (CC BY 4.0)] 


\section{BACKGROUND}

Gestational diabetes mellitus (GDM) is a major health problem arising due to insulin resistance in pregnant women. There is an increase in its incidence over the past decade owing to the sedentary lifestyle practices. It is associated with multiple maternal complications which may cause end organ failure, complicated labour and delivery and thereby increasing the maternal morbidity and mortality. The foetus is also at risk for problems beginning from in utero and extending into the neonatal period and adult life. This is the reason for obstetricians and endocrinologists to have an extra eye on patients who are prone to develop gestational diabetes mellitus. Hence, screening for gestational diabetes mellitus is more important than diagnosing it later in the course of antenatal period. Initially screening was opted only for women with risk factors but currently it is done for all as a large proportion of women develop gestational diabetes mellitus despite having no risk factors. Screening techniques vary from obstetrician and the most accepted methods are the non challenge glucose test or the glucose challenge tests like the American Diabetic Association (ADA) ${ }^{1}$ which approved 3 hours 100gm oral glucose tolerance test (OGTT) for the diagnosis of GDM, it has recently included in its recommendations the use of 2 hours 75 gms oral glucose tolerance test. WHO panel although in general maintaining previous diagnosis recommendations, now characterizes gestational diabetes mellitus as the joint category of diabetes and impaired glucose tolerances and they have adopted in pregnancy the same diagnostic criteria applied to a $2 \mathrm{hr}$ $75 \mathrm{gms}$. Oral Glucose tolerance test in non pregnant women. (IGT - 2 hr PPG 140 - $200 \mathrm{mg} / \mathrm{dl}$ ). The pregnant women who meet the WHO criteria for impaired glucose test (IGT) are classified as having gestational diabetes mellitus. Using 2 hours' plasma glucose $>140 \mathrm{mg} / \mathrm{dl}$ as a one step procedure is simple and economical particularly for the countries ethnically more prone to high prevalence of diabetes. There is a tendency all over the world to accept the $75 \mathrm{gms}$ oral glucose tolerance test as a universal test for screening. Glycosylated haemoglobin ( $\mathrm{HbA1c}$ ) is an evolving tool in the world of diabetes but its significance in screening for gestational diabetes mellitus is still a mystery. Studies have been made to assess if glycosylated haemoglobin values of 5.7 - $6.4 \%$ which has been considered as impaired glucose tolerance in non obstetric population can be used as a cut off for the obstetric population. When blood glucose levels are high, the glucose molecules attach itself to the haemoglobin in red blood cells. The longer the hyperglycaemia persists in blood, the more the glucose binding to haemoglobin in the red blood cells and higher the glycosylated haemoglobin. The average level of glycosylated haemoglobin is seen in red blood cells life cycle and changes accordingly. Haemoglobin molecule undergoes glycosylation and cannot be changed. Glycosylated Haemoglobin is used to assess the long term effectiveness of serum glucose regulation. HbA1c is the average blood glucose level of red blood cells during its lifetime. The life time of RBC is 117 days for men and 106 days for women. The glucose levels which are done on days next to the test have more levels than those done on days far away from the test. Glycosylated haemoglobin test is done to check the glycaemic control in women who might be having risk factors and in those who may be pre-diabetic. Monitoring and achieving the target glycaemic control in diabetic patients. Prenatal counselling of women with diabetes. The NICE guidelines for diabetes in pregnancy state that women with diabetes should aim to achieve HbA1c result of $6.1 \%$ or lower. If the target is achieved it will help to minimise the congenital malformations. If the woman has HbA1c above $10 \%$, it is strongly advised to avoid pregnancy until good diabetic control is achieved and sustained. Glycosylated haemoglobin as a screening tool for gestational diabetes mellitus and also as a target scale in monitoring of glycaemic control is still a matter of debate with several ambiguous studies. Long term vascular complications like coronary artery diseases, stroke, peripheral neuropathy especially loss of sensation in the feet, heart attack, blindness, kidney failure, heart failure, gastroparesis which is slow emptying of the stomach, and gangrene are seen in those with persistent elevations in blood sugar levels. Wound healing is also delayed because of poor glycaemic control which is a shortterm complication. Glucose-6-phosphate dehydrogenase deficiency, any condition causing premature red blood cell death and sickle cell disease have shortened lifespan of red blood cells and therefore low levels of HbA1c. Further more if a woman has undergone blood transfusion there will be rapid replacement of the lost red blood cells with the newly formed red blood cells and these newly formed cells will lead to low levels of glycosylated haemoglobin and hence the newly formed red blood cells will lead to underestimation of actual average levels. People with Vitamin B12 deficiency and folate deficiency have longer lifespan of red blood cells than others and hence have higher than the expected values of glycosylated haemoglobin. If there was a blood transfusion previously about two months prior, there will be a distortion in the value of glycosylated haemoglobin resulting in overestimation of actual average blood glucose levels due to abnormal synchronisation of the age of the red blood cells, which further results in older than normal average blood cell life.

\section{METHODS}

It is a prospective observational study. This study was conducted from August 2015 to August 2017. All women of gestational age 6 to 12 weeks who came to tertiary care institute were taken for this study. Informed consent was obtained and a detailed history taking was done as per proforma. Along with routine antenatal investigations, glycosylated haemoglobin (HbA1C) was also done. Oral Glucose Tolerance Test (OGTT) was to be performed at 24 28 weeks as per the World Health Organisation - 75gm criteria and the results were tabulated and analysed to know the significance of glycosylated haemoglobin (HbA1C) in the outcome of gestational diabetes mellitus. Further fasting and postprandial blood sugar tests were done in the $3^{\text {rd }}$ trimester for all patients as a secondary screening tool for gestational diabetes mellitus. Secondary outcomes of obstetric and neonatal complications were also studied.

\section{Inclusion Criteria}

- Antenatal women of gestational age 6 to 12 weeks. All the pregnant women gave informed consent for the study. 


\section{Exclusion Criteria}

- Overt diabetic

- HbA1c value $>6.4 \%$

\section{Statistical Analysis}

The P value was calculated by chi-square Test. The Statistical software used was SPSS-16- Statistical Package for Software Solution.

\section{RESULTS}

\begin{tabular}{|c|c|c|c|}
\hline $\begin{array}{l}\text { Glycosylated } \\
\text { Haemoglobin } \\
\text { HbA1c } \\
(\mathrm{N}=323)\end{array}$ & $\begin{array}{l}2^{\mathrm{ND}} \\
\text { Trimester } \\
(\mathrm{N}=38)\end{array}$ & $\begin{array}{l}3^{\text {RD }} \text { Trimester } \\
\quad(\mathrm{N}=33)\end{array}$ & $\begin{array}{l}\text { Total Gestational } \\
\text { Diabetes Mellitus } \\
\qquad(\mathrm{N}=71)\end{array}$ \\
\hline $4.0-4.5(\mathrm{~N}=50)$ & - & $2(2.8 \%)$ & $2(2.8 \%)$ \\
\hline $4.6-5.0(\mathrm{~N}=148)$ & 7 (9.9 \%) & $9(12.7 \%)$ & $16(12.7 \%)$ \\
\hline $5.1-5.6(\mathrm{~N}=106)$ & $23(32.4 \%)$ & $16(22.5 \%)$ & $39(54.9 \%)$ \\
\hline $5.7-6.4(\mathrm{~N}=19)$ & $8(11.3 \%)$ & $6(8.5 \%)$ & $14(19.7 \%)$ \\
\hline \multicolumn{4}{|c|}{$\begin{array}{c}\text { Table 1. Total Number of Patients with Glycosylated Haemoglobin } \\
\text { HbA1c in the } 2^{\text {nd }} \text { and } 3^{\text {rd }} \text { Trimester and Total Number of Patients with } \\
\text { Gestational Diabetes Mellitus }\end{array}$} \\
\hline
\end{tabular}

71 patients $(21.9 \%)$ were diagnosed with gestational diabetes mellitus.

\begin{tabular}{|cccc|}
\hline Treatment & $\begin{array}{c}\text { HbA1c }<\mathbf{5 . 7} \\
\mathbf{\%}(\mathbf{N}=\mathbf{5 7})\end{array}$ & $\begin{array}{c}\text { HbA1c 5.7 - 6.4 } \\
\mathbf{\%}(\mathbf{N}=\mathbf{1 4})\end{array}$ & $\begin{array}{c}\text { Total } \\
\text { GDM }(\mathbf{N}=\mathbf{7 1})\end{array}$ \\
Diet & 31 & 3 & $34(47.8 \%)$ \\
OHA & 22 & 6 & $28(39.4 \%)$ \\
Insulin & 2 & 1 & $3(4.2 \%)$ \\
OHA + Insulin & 2 & 4 & $6(8.4 \%)$ \\
\hline \multicolumn{4}{|r|}{ Table 2. Gestational Diabetes Mellitus and Treatment } \\
\hline
\end{tabular}

- Diet modification was required for majority of patients (47.8\%).

- Oral hypoglycaemic drugs were required by 28 patients (39.4\%).

- Insulin was necessary for glycaemic control in 3 patients (4.2\%).

- Oral hypoglycaemic drugs with insulin was required in 6 patients $(8.4 \%)$.

- 29 patients (40.8\%) had a spontaneous vaginal delivery.

- 4 patients (5.6\%) had assisted vaginal delivery.

- 38 patients (53.5\%) had lower segment caesarean section.

\begin{tabular}{|cccc|}
\hline Mode of Delivery & $\begin{array}{c}\text { Normal } \\
\text { Glycosylated } \\
\text { Haemoglobin } \\
\text { HbA1c(N =57) }\end{array}$ & $\begin{array}{c}\text { Increased } \\
\text { Glycosylated } \\
\text { Haemoglobin } \\
\text { HbA1c(N =14) }\end{array}$ & $\begin{array}{c}\text { Total Gestational } \\
\text { Diabetes } \\
\text { Mellitus(N =71) }\end{array}$ \\
$\begin{array}{c}\text { Spontaneous vaginal } \\
\text { delivery }\end{array}$ & 21 & 8 & $29(40.8 \%)$ \\
$\begin{array}{c}\text { Assisted vaginal delivery } \\
\text { Lower segment } \\
\text { caesarean section }\end{array}$ & 4 & - & $4(5.6 \%)$ \\
\hline \multicolumn{4}{|c|}{ Table 3. Mode of Delivery in Patients with } \\
Gestational Diabetes Mellitus
\end{tabular}

\begin{tabular}{|cc|}
\hline Maternal Complications & Total \\
Shoulder dystocia & 1 \\
Perineal tear & 2 \\
Perinatal Complication & 16 \\
NICU admissions & 2 \\
Macrosomia & - \\
Congenital anomalies & 9 \\
Foetal hypoglycaemia & - \\
\hline Stillbirth & Table 4. Maternal and Neonatal Complications in $\mathbf{G D M}$ \\
\hline
\end{tabular}

Most of the babies required Neonatal Intensive Care Unit.

\begin{tabular}{|cccc|}
\hline $\begin{array}{c}\text { Glycosylated } \\
\text { Haemoglobin } \\
\text { (HbA1c) }\end{array}$ & $\begin{array}{c}\text { Sample } \\
(\mathbf{N}=\mathbf{3 2 3})\end{array}$ & $\begin{array}{c}\text { Gestational Diabetes } \\
\text { Mellitus(N =71) }\end{array}$ & $\begin{array}{c}\text { \% of Gestational } \\
\text { Diabetes Mellitus }\end{array}$ \\
\hline$<5.7$ & 304 & 57 & $18.7 \%$ \\
$5.7-6.4$ & 19 & 14 & $73.6 \%$ \\
\hline Table 5. Correlation between Glycosylated Haemoglobin \\
and Gestational Diabetes Mellitus
\end{tabular}

$73.6 \%$ of patients from the study population with elevated glycosylated haemoglobin of 5.7 - $6.4 \%$ had gestational diabetes mellitus.

\section{DISCUSSION}

Glycosylated haemoglobin has been well accepted in the nonobstetric population as a tool for diagnosis of diabetes. In normal individuals glycosylated haemoglobin ( $\mathrm{HbA1c}$ ) value of $5.7-6.4 \%$ indicates impaired glucose tolerance as well as a high risk of developing diabetes in the future. Our study is aimed to correlate early HbA1c levels with the subsequent development of gestational diabetes mellitus, with the hypothesis that patients with the value of $5.7-6.4 \%$ are at greater risk of developing gestational diabetes mellitus than those with a level $<5.7 \%$. In our study, glycosylated haemoglobin was done in the first trimester and further diagnosed gestational diabetes mellitus based on the WHO criteria of $2 \mathrm{hr}$ PPBS $\geq 140 \mathrm{mg} / \mathrm{dl}$ in second trimester and PPBS in third trimester. No correlation was seen in the trimester of diagnosis of gestational diabetes mellitus with glycosylated haemoglobin. Similarly, Alex Fong et al. $(2014)^{2}$ in his retrospective study of 526 women who underwent $\mathrm{HbA1c}$ in the first trimester followed by oral glucose tolerance test in the second trimester. The study showed that there was no correlation in the trimester of diagnosis with $\mathrm{HbA1c}$ values but a significance was noted in the fasting glucose tolerance value with glycosylated haemoglobin (P value $<0.001$ ) Aldasouqi et al. $(2008)^{3}$ in their retrospective study of 145 eligible patients found gestational diabetes mellitus in 124 patients with oral glucose tolerance test. The sensitivity percentage of patients with glycosylated haemoglobin (HbA1c) values equal to or above sequential cut-off values of $5.0 \%, 5.5 \%, 6.0 \%, 6.5 \%$, and 7.0 $\%$ were $100 \%, 98.4 \%, 87.1 \%, 62.9 \%$, and $39.5 \%$, respectively. The mean glycosylated haemoglobin (HbA1c) of the patients with gestational diabetes mellitus was $6.9 \pm 0.8$ $\%$ compared with $6.4 \pm 0.6 \%$ for those without gestational diabetes mellitus.

At an arbitrary glycosylated haemoglobin (HbA1c) cut-off value of $6.0 \%$ nearly $87.1 \%$ of patients could have been diagnosed with gestational diabetes mellitus. This study suggested that glycosylated haemoglobin (HbA1c) is a reasonably sensitive screening measure for gestational diabetes mellitus in this high-risk population. Balaji et al. (2007) ${ }^{4}$ established the normal mean glycosylated haemoglobin (HbA1c) values in Asian - Indian pregnant women as $5.36 \pm 0.36 \%$. They also found that the mean glycosylated haemoglobin (HbA1c) level in women with gestational diabetes mellitus at diagnosis during different trimesters was $6 \%$.

Rajesh Rajput et al. (2012) ${ }^{5}$ conducted a study with 607 patients in 2012, the values of glycosylated haemoglobin (HbA1c) ranged from 4.0 - $6.1 \%$. Gestational diabetes 
mellitus was diagnosed by International association of diabetes and pregnancy study groups (IADPSG). The mean standard deviation glycosylated haemoglobin (HbA1c) value in women with gestational diabetes mellitus was $5.73 \pm 0.34$ $\%$, while it was $5.34 \pm 0.35 \%$ in women without gestational diabetes mellitus. The difference in the two HbA1c values was found to be statistically significant.

It was observed that a glycosylated haemoglobin (HbA1c) cut-off value of $5.95 \%$ had a sensitivity of $28.6 \%$ and a specificity of $97.2 \%$ in diagnosing gestational diabetes mellitus whereas a glycosylated haemoglobin (HbA1c) cut-off value of $5.45 \%$ had a sensitivity of $85.7 \%$ and a specificity of $61.1 \%$. Using glycosylated haemoglobin (HbA1c) as the initial test, if the level of HbA1c is $\geq 5.95 \%$, then the woman can be labelled as having gestational diabetes mellitus. If the cut off glycosylated haemoglobin (HbA1c) value is $5.95 \%$ then it has a specificity of $>97 \%$ in identifying cases of gestational diabetes mellitus. Ingrid Hov Odsaeter et al. ${ }^{6}$ conducted a study on 855 women in whom World Health Organisation / International association of diabetes and pregnancy study groups (IADPSG) ${ }^{7}$ was used to diagnose gestational diabetes mellitus along with $\mathrm{HbA1c}$ values done at 18 - 22 and 32 - 36weeks.

The study concluded that around $30 \%$ of pregnant women could have potentially avoided an oral glucose tolerance test by using glycosylated haemoglobin (HbA1c) $\leq 4.8 \%$ to exclude GDM - IADPSG with a sensitivity of $88 \%$ at pregnancy weeks $18-22$ and by using glycosylated haemoglobin $(\mathrm{HbA} 1 \mathrm{c}) \leq 5.0 \%$ with a sensitivity of $97 \%$ at pregnancy weeks 32 - 36. Further, $16 \%$ could have potentially avoided an oral glucose tolerance test with a sensitivity of $96 \%$ by using HbA1c $\leq 4.7 \%$ at pregnancy weeks $18-22$ to exclude GDM -IADPSG ${ }^{7}$ throughout pregnancy.

Adding other variables to predict gestational diabetes mellitus did not significantly increase the number of potentially avoidable oral glucose tolerance test. HbA1c was not accurate at diagnosing GDM - IADPSG ${ }^{7}$ since most of those diagnosed would have been false positives.

Glycosylated Haemoglobin (HbA1c) was inaccurate at screening for GDM - WHO at any time point. Neither HbA1c nor gestational diabetes mellitus was accurate in predicting preeclampsia or birth weight. Our study showed no significance between glycosylated haemoglobin and type of medical treatment or rate of caesarean delivery. Birth weight had significance with glycosylated haemoglobin in our study and the same was seen with Ingrid Hov Odsaeter et al. (2016). ${ }^{6}$ Alex Fong et al. $(2014)^{2}$ showed no statistical significance in the type of treatment, mode of delivery and birth weight. Although the overall diagnostic accuracy for $\mathrm{HbA1c}$ in diagnosing gestational diabetes mellitus by IADPSG / World Health Organisation criteria was similar for the present and previous studies, the diagnostic thresholds for glycosylated haemoglobin (HbA1c) and the sensitivity and specificity at the same levels of $\mathrm{HbA1c}$ differ between studies. $^{7}$ These differences probably reflect diversities between populations, testing at different gestational lengths and the analytical methods used.

\section{Summary}

In the present study a total of 323 patients were screened with first trimester glycosylated haemoglobin (HbA1c) and followed till delivery. Only $21.9 \%$ developed gestational diabetes mellitus. Elevated HbA1c value of $5.7-6.4 \%$ was seen in $5.9 \%$. Among the patients with elevated glycosylated haemoglobin (HbA1c) of $5.7-6.4 \%, 73.6 \%$ of them developed gestational diabetes mellitus.

Gestational diabetes mellitus was diagnosed in $54 \%$ of patients during the second trimester and $46 \%$ of patients in the third trimester. Family history of diabetes was equivocal as $47.8 \%$ of patients with gestational diabetes mellitus had either parents being diabetic whereas $52.1 \%$ of patients had neither of the parents diabetic.

$47.8 \%$ patients had good glycaemic control with just diet. $89.1 \%$ patients delivered at term and $16.8 \%$ patients delivered preterm. $46.2 \%$ patients delivered by Normal Vaginal delivery and $53.5 \%$ delivered by Lower Segment Caesarean Section. $22.5 \%$ of babies were admitted in the Neonatal Intensive Care Unit for preterm care, hypoglycaemia, macrosomia, and other reasons. Foetal hypoglycaemia (56.2 \%) was the commonest reason for admission in patients with gestational diabetes mellitus.

\section{CONCLUSIONS}

Most of the patients with elevated glycosylated haemoglobin in the first trimester had high chance of developing gestational diabetes mellitus. With the increasing incidence of gestational diabetes mellitus, it is important to improvise on our screening methods and also consider screening the entire population and not restrict it to a certain group of high risk patients, as quite a number of patients are being undiagnosed leading to an increase in maternal and foetal morbidity and mortality. Glycosylated haemoglobin is indeed a simple way of screening, but its solitary use remains controversial and hence could possibly be tried as an adjunct with other screening methods.

Data sharing statement provided by the authors is available with the full text of this article at jemds.com.

Financial or other competing interests: None.

Disclosure forms provided by the authors are available with the full text of this article at jemds.com.

\section{REFERENCES}

[1] American Diabetes Association. Diagnosis and classification of diabetes mellitus. Diabetes Care 2007;30(Suppl 1):S42-7.

[2] Fong A, Serra AE, Gabby L, et al.Use of hemoglobin $A_{1 c}$ as an early predictor of gestational diabetes mellitus. Am J Obstet Gynecol 2014;211(6):641.e1-7.

[3] Aldasouqi SA,Soloman DJ, Bokhari SA, et al. Glycohemoglobin A1c: a promising screening tool in gestational diabetes mellitus. Int J Diabetes Dev Ctries 2008;28(4):121-4.

[4] Balaji V, Madhuri BS, Ashalatha S, et al. A1C in gestational diabetes mellitus in Asian Indian women. Diabetes Care 2007;30(7):1865-7. 
[5] Rajput R, Yadav Y, Rajput M, et al. Utility of HbA1c for diagnosis of gestational diabetes mellitus. Diabetes Res Clin Pract 2012;98(1):104-7.

[6] Odsaeter IH, Asberg A, Vanky E, et al. HbA1c as screening for gestational diabetes mellitus in women with polycystic ovary syndrome. BMC Endocr Disord 2015;15:38.
[7] Metzger BE, Gabbe SG, Persson B, et al. International association of diabetes and pregnancystudy groups recommendations on the diagnosis and classification of hyperglycemia in pregnancy. Diabetes Care 2010;33(3):676-82. 